\title{
Title
}

\section{Maternal serum laeverin (aminopeptidase Q) measured in the first trimester of pregnancy does not predict preeclampsia}

\section{Authors}

Kasper Pihl ${ }^{1}$ (ORCID 0000-0003-1592-3388), Steen Sørensen² (ORCID 0000-0002-9766-

5820), Mona Nystad ${ }^{3}$ (ORCID 0000-0002-0555-6951), Ganesh Acharya ${ }^{3,4}$ (ORCID 00000002-1997-3107) \& Finn Stener Jørgensen¹ (ORCID 0000-0002-1592-5407)

${ }^{1}$ Fetal Medicine Unit, Department of Obstetrics and Gynecology, Copenhagen University Hospital Hvidovre, Denmark

${ }^{2}$ Department of Clinical Biochemistry, Copenhagen University Hospital Hvidovre, Denmark

${ }^{3}$ Women's Health and Perinatology Research Group, Department of Clinical Medicine, UiTThe Arctic University of Norway and University Hospital of North Norway, Tromsø, Norway

${ }^{4}$ Division of Obstetrics and Gynecology, Department of Clinical Science, Intervention and Technology, Karolinska Institute, Stockholm, Sweden

\section{Corresponding author}

Kasper Pihl, MD, PhD

Fetal Medicine Unit, Department of Obstetrics \& Gynecology

Copenhagen University Hospital Hvidovre

Kettegård Allé 30, DK-2650 Hvidovre, Denmark

E-mail: Kasper_Pihl@dadlnet.dk

Mobile: +4538621408 


\section{Short title}

Serum laeverin

\section{Keywords}

- Laeverin

- Aminopeptidase Q

- Preeclampsia

- Serum

- First trimester

\section{Count}

Manuscript word: 1.661

Tables: 2

Figures: 2

\section{Abbreviations}

PE - Preeclampsia

MoM - Multiple of the median 


\begin{abstract}
Objective

The aim of this study was to compare the laeverin level in maternal serum from first trimester (11-14 weeks) of pregnancy between normal pregnancies and pregnancies that later developed preeclampsia (PE).
\end{abstract}

\title{
Material and methods
}

This was a case-cohort study. The laeverin concentration was measured in cases with preterm PE $(n=55)$, term PE $(n=95)$ and a reference group of randomly selected women with normal pregnancy outcome $(n=200)$ in stored serum samples collected from the double-test as part of the combined first trimester trisomy 21 screening program. The samples were thawed and analyzed for laeverin. The median gestational age at blood sampling was 77 days (range 5796 days). Multiple regression analysis was performed to establish a normal median. Concentrations were converted to multiples of the median (MoM) and groups were compared using the Mann-Whitney U-test.

\section{Results}

In the reference group laeverin was significantly correlated with gestational age $(r=0.18$, $\mathrm{p}=0.01)$ and its concentration ranged from $41-393 \mu \mathrm{g} / \mathrm{L}$. No significant differences in the median laeverin MoM were found between the reference group (1.01 MoM) and cases with preterm PE (0.98 MoM) or term PE (0.96 MoM).

\section{Conclusion}

First trimester maternal serum laeverin level cannot be used to predict preeclampsia. 


\section{Introduction}

Preeclampsia (PE) affects around 3-5\% of pregnancies and is a major cause of maternal and perinatal mortality and morbidity [1]. The pathogenesis of PE is not fully understood, but placental and endothelial dysfunction seem to play important roles [2]. Several biomarkers have been suggested to predict PE, but no individual marker with sufficient specificity and sensitivity has been identified. Search for an appropriate biomarker that could accurately predict the development of this placenta-specific disorder is ongoing.

Laeverin, also known as aminopeptidase Q, is a placenta specific membrane-bound aminopeptidase. The protein was first reported by Fujiwara et al. in 2004 and was found to be expressed by the human trophoblast cells [3]. Laeverin is suggested to play a role in placentation by a regulating molecular mechanism associated with extravillous trophoblast invasion. Laeverin expression is detectable from very early stage of placentation at around four weeks of gestation [4].

It has been suggested that laeverin may have a role in the pathogenesis of PE [5]. This is supported by the finding of laeverin gene being significantly upregulated (10 fold) in placentas from pregnancies complicated by severe PE [6]. Furthermore, in the second trimester, maternal plasma laeverin level was found to be reduced in pregnancies that later developed PE [7].

We hypothesized that laeverin might be altered in the first trimester maternal serum in pregnancies who later develop PE. The aim of this study was to compare the first trimester maternal serum laeverin concentrations between normal pregnancies and pregnancies that 
were complicated by PE. Furthermore, we wanted to explore potential associations to figure out whether first trimester laeverin could be used as a screening test for PE. 


\section{Material \& Methods}

\section{Study population}

This study was conducted on a cohort of nulliparous women with singleton pregnancies consecutively enrolled into the first trimester combined screening program for trisomy 21 in the period 1 January 2005 to 31 December 2010 at Copenhagen University Hospital Hvidovre, Denmark. Data were obtained from the local fetal medicine database (Astraia software, München, Germany) and the local obstetric database. The SPSS sample function (Mersenne Twister random number generator) was used for random selection of 200 reference cases from the cohort restricted to those with delivery at $\geq 37$ weeks +0 days, a birth weight appropriate for gestational age $( \pm 2 \mathrm{SD})[8]$ and no registered diagnosis of $\mathrm{PE}$. In pregnancies with a registered diagnosis of $\mathrm{PE}$, the diagnosis was validated by scrutinization of the medical records to ensure that the diagnostic criteria were fulfilled. In total, 100 of 237 cases with term PE were selected randomly with the SPSS sample function. All cases with preterm PE were included $(\mathrm{n}=61)$.

The study was approved by the Danish Research Ethics Committee (H-1-2012-097) and the Data Protection Agency in Denmark (01820 HVH-2012-035).

\section{Serum sample analysis}

The blood samples were collected as part of the first trimester combined screening program for trisomy 21 between 8 and 14 weeks of gestation. Serum samples were retrieved from storage, thawed and analyzed for laeverin. It was not possible to localize 11 samples from storage-freezer in cases with PE. The concentration of laeverin (aminopeptidase Q) was measured using a commercial enzyme-linked immunosorbent assay (ELISA) (EIAab Science 
Co Ltd., Wuhan, China). The measurement was performed in duplicate according to the manufacturer's specifications. A parallelism was observed between the laeverin standard curve and two serum samples diluted with sample diluent 1:25, 1:50, 1:100 and 1:200 (Figure 1). A serum sample dilution of 1: 50 was chosen to cover the concentration range 20-500 $\mu \mathrm{g} / \mathrm{L}$ laeverin. The inter-assay variation was $14.0 \%$ at $133 \mu \mathrm{g} / \mathrm{L}$ and $21.6 \%$ at $14 \mu \mathrm{g} / \mathrm{L}(\mathrm{n}=11)$. The lower detection limit was $0.05 \mu \mathrm{g} / \mathrm{L}$.

\section{Outcome measures}

PE was defined as gestational hypertension with proteinuria according to the International Society for the Study of Hypertension in Pregnancy (ISSHP) [9]. Gestational hypertension was defined as either a systolic blood pressure exceeding $140 \mathrm{mmHg}$ or a diastolic bloodpressure exceeding $90 \mathrm{mmHg}$ (based on two measurements taken at least four hours apart) in a previously normotensive woman after 20 weeks of gestation. The criteria for proteinuria were $\geq 300 \mathrm{mg} / 24$ hours or ' $2+$ ' on urine dipstick testing. PE cases were stratified into preterm PE (delivery $<37$ weeks +0 days) and term PE (delivery $\geq 37$ weeks +0 days). Small-for-gestational age (SGA) was defined as a birthweight deviation $\geq 2$ standard deviations from the mean birthweight in normal pregnancies according to gestational age [8].

\section{Statistical analyses}

Data distribution was checked for normality using the Shapiro-Wilk test, and log-transformed to achieve normal distribution if required. Multiple regression analysis was performed to establish a normal median for laeverin in the reference group. Laeverin concentrations were then transformed to multiples of the median $(\mathrm{MoM})$ values in both cases and references. Groups were compared using the Mann-Whitney U-test or the Chi-Square test as appropriate. A p-value $<0.05$ was considered significant. The statistical software packages SPSS 22.0 
(SPSS Inc., Chicago, IL, USA) was used for the data analyses. 


\section{Results}

The maternal characteristics for each outcome group are outlined and compared in Table 1. The body mass index (BMI) was significantly elevated in pregnancies with PE. In the reference group a positive correlation between the laeverin concentration (log transformed) and the gestational age was found $(\mathrm{r}=0.18, \mathrm{p}=0.01)$. The laeverin concentration ranged from $41 \mu \mathrm{g} / \mathrm{L}$ to $393 \mu \mathrm{g} / \mathrm{L}$ in the reference group. Figure 2 shows the laeverin concentration according to gestational age in the different outcome groups. In a multivariate regression analysis of the reference group, the gestational age and maternal weight contributed significantly to the model describing the normal median for laeverin as $\mu \mathrm{g} / \mathrm{L}=10 \mathrm{exp}$ $\left(0.005^{*}\right.$ gestational age*days ${ }^{-1}+0.003 *$ maternal weight* $\left.\mathrm{kg}^{-1}+1.925\right)$. The laeverin MoM medians in the outcome groups are outlined in Table 2. No significant difference was found between the reference group and cases with PE. A further stratification of cases with PE into preterm and term $\mathrm{PE}$, and the fetus being small-for-gestational age or not, showed no significant change in the laeverin MoM median. 


\section{Discussion}

To the best of our knowledge this is the first study reporting first trimester maternal serum laeverin concentrations in pregnancies that were complicated by PE later compared to normal pregnancies. We found no significant difference in the laeverin MoM values between the PE and the reference groups. We had expected an altered level of laeverin in cases with preterm PE, because of the known strong association with impaired placentation in this group and the reported significance of laeverin in the early process of placentation $[4,10]$. However, the laeverin levels were also similar between the preterm and the term PE groups.

We found a positive correlation between maternal serum laeverin concentration and gestational age in the late first trimester, which is in contrast to the finding of negative correlation reported in the second and third trimester [7]. However, the median laeverin concentrations measured during 8-14 weeks were higher compared to the values reported during 22-40 weeks. Although the differences in the study design (longitudinal vs. crosssectional) and the samples used for measuring laeverin (plasma vs. serum) may explain some variability, this is an interesting finding. Higher and increasing concentration in the first trimester may suggest that laeverin has an important role to play in the first trimester placentation process. Expression studies using first trimester placental samples and maternal blood obtained simultaneously, such as during termination of pregnancy, could provide more insight into the relationship between placental and maternal compartments.

Maternal serum laeverin might be a biomarker of PE in the second and third trimester. In a recent study, Nystad et al.[7] report that second trimester maternal plasma laeverin was 
significantly reduced in pregnancies that developed PE. It was speculated that the reduction in laeverin during the development of PE was caused by trapping of laeverin in the placenta or in microvesicles in the fetal capillaries [5]. However, the study was based on only a few cases with PE $(n=15)$ and therefore needs to be confirmed in larger series.

The strength of our study is the inclusion of large number of cases with PE with validated diagnosis. It has previously been reported that Danish registry data might be restricted due to variability in the diagnostic criteria and reporting procedures for $\mathrm{PE}$, which emphasizes the need for clinical validation $[11,12]$. We acknowledge that our sample size may not be large enough to detect a small difference in the laeverin concentration between cases and the reference group. However, such a small difference is unlikely to be relevant in the context of screening for PE.

In conclusion, first trimester maternal serum laeverin is not altered in pregnancies that are complicated by PE later in gestation compared to normal pregnancies. Maternal serum laeverin measured in the first trimester does not appear to predict PE and is therefore not a useful marker in first trimester screening for PE. 


\section{Acknowledgement}

We want to thank the laboratory technicians, Vibeke Myrhøj and Bente Madsen, Department of Clinical Biochemistry, Copenhagen University Hospital Hvidovre, Denmark, for providing stored serum samples for the study and performing the laeverin analysis of the samples, respectively.

\section{Funding}

The study has received financial support from Axel Muusfeldts Foundation, Aase \& Ejnar Danielsens Foundation and Hans \& Nora Buchards Foundation.

\section{Declaration of interests}

The authors report no conflict of interest 


\section{References}

1. Mol BWJ, Roberts CT, Thangaratinam S, Magee LA, de Groot CJM, Hofmeyr GJ. Preeclampsia. Lancet 2016;387:999-1011.

2. Redman CW, Sargent IL, Staff AC. IFPA Senior Award Lecture: making sense of preeclampsia - two placental causes of preeclampsia? Placenta 2014;35:S20-25.

3. Fujiwara H, Higuchi T, Yamada S, Hirano T, Sato Y, Nishioka Y, et al. Human extravillous trophoblasts express laeverin, a novel protein that belongs to membrane-bound gluzincin metallopeptidases. Biochem Biophys Res Commun 2004;313:962-8.

4. Horie A, Fujiwara H, Sato Y, Suginami K, Matsumoto H, Maruyama M, et al. Laeverin/aminopeptidase $\mathrm{Q}$ induces trophoblast invasion during human early placentation. Hum Reprod 2012;27:1267-76.

5. Nystad M, Sitras V, Larsen M, Acharya G. Placental expression of aminopeptidase-Q (laeverin) and its role in the pathophysiology of preeclampsia. Am J Obstet Gynecol 2014;211:686.e1-31.

6. Sitras V, Paulssen R, Leirvik J, Vårtun A, Acharya G. Placental gene expression profile in intrauterine growth restriction due to placental insufficiency. Reprod Sci 2009;16:701-11.

7. Nystad M, Sitras V, Flo K, Widnes C, Vårtun Å, Wilsgaard T, et al. Longitudinal reference 
ranges for maternal plasma laeverin, and its role as a potential biomarker of preeclampsia. BMC Pregnancy Childbirth 2016;16:377.

8. Marsal K, Persson PH, Larsen T, Lilja H, Selbing A, Sultan B. Intrauterine growth curves based on ultrasonically estimated foetal weights. Acta Paediatr 1996;85:843-8.

9. Tranquilli AL, Dekker G, Magee L, Roberts J, Sibai BM, Steyn W, et al. The classification, diagnosis and management of the hypertensive disorders of pregnancy: A revised statement from the ISSHP. Pregnancy Hypertens 2014;4:97-104.

10. Fujiwara H. Membrane-bound peptidases regulate human extravillous trophoblast invasion. Placenta 2007;28:S70-75.

11. Klemmensen AK, Olsen SF, Wengel CM, Tabor A. Diagnostic criteria and reporting procedures for pre-eclampsia: a national survey among obstetrical departments in Denmark. Eur J Obstet Gynecol Reprod Biol 2005;123:41-5.

12. Klemmensen AK, Olsen SF, Osterdal ML, Tabor A. Validity of preeclampsia-related diagnoses recorded in a national hospital registry and in a postpartum interview of the women. Am J Epidemiol 2007;166:117-24. 


\section{Table 1.}

Maternal characteristics with values in median (range) and $\mathrm{n}(\%)$

\begin{tabular}{lccc}
\hline & $\begin{array}{c}\text { Reference group } \\
(\boldsymbol{n}=\mathbf{2 0 0})\end{array}$ & Term PE (n=95) & Preterm PE \\
& $29.0(16-40)$ & $28.0(19-39)^{*}$ & $29.0(18-44)$ \\
\hline Age (years) & $21.9(15.9-39.8)$ & $23.5(17.0-$ & $22.6(17.0-$ \\
Pre-pregnancy BMI $\left(\mathrm{kg} / \mathrm{m}^{2}\right)$ & $179(89.5)$ & $85(89.5)$ & $53.1)^{*}$ \\
Caucasian ethnicity & $14(7.0)$ & $6(6.3)$ & $5(90.9)$ \\
Cigarette smoker & $77(57-96)$ & $77(57-94)$ & $77(57-96)$ \\
Gestaional age at blood sampling & & \\
$($ days $)$ & & \\
\hline${ }^{*}$ p $<0.05$ (reference group vs outcome group, Mann-Whitney U-test) &
\end{tabular}


Table 2.

Laeverin MoM values in different outcome groups

\begin{tabular}{|c|c|c|c|}
\hline Outcome group & Median & $I Q R$ & $p$-value $e^{a}$ \\
\hline Reference group $(n=200)$ & 1.01 & 0.56 & - \\
\hline $\mathrm{PE}(\mathrm{n}=150)$ & 0.98 & 0.67 & 0.535 \\
\hline - Term PE $(n=95)$ & 0.96 & 0.61 & 0.725 \\
\hline - Preterm PE $(n=55)$ & 0.98 & 0.79 & 0.483 \\
\hline$-P E+\operatorname{SGA}(n=27)^{b}$ & 1.02 & 0.79 & 0.702 \\
\hline
\end{tabular}

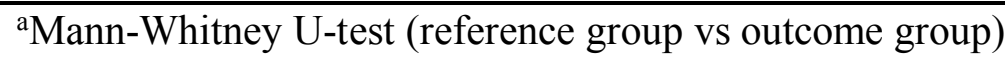

${ }^{\mathrm{b}}$ Cases with SGA in all PE cases 


\section{Legends}

\section{Figure 1.}

Laeverin standard curve and two serum samples diluted 1:25, 1:50, 1:100 and 1:200.

\section{Figure 2.}

Maternal serum laeverin concentration according to gestational age in different outcome groups. 


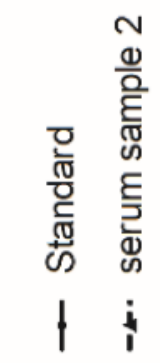

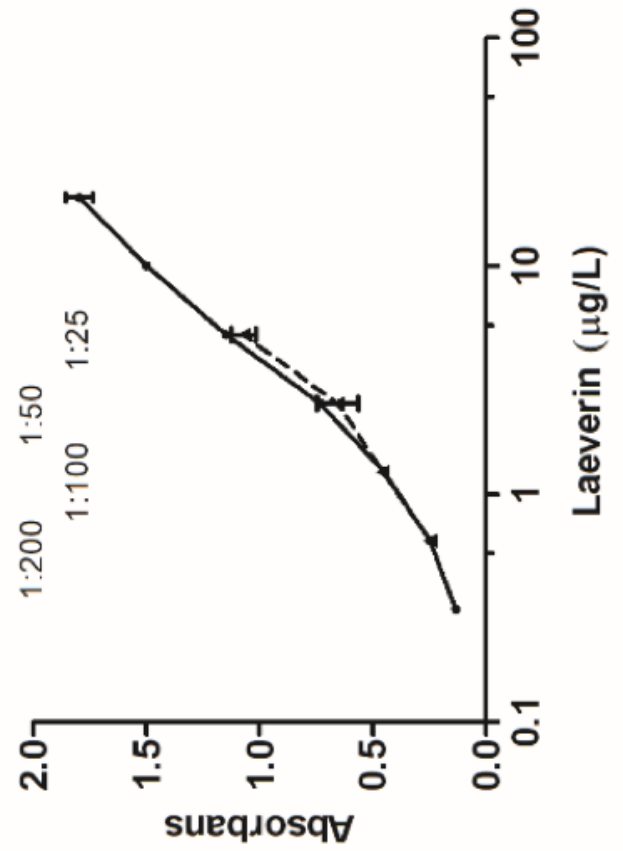

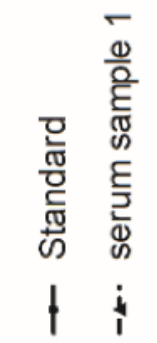

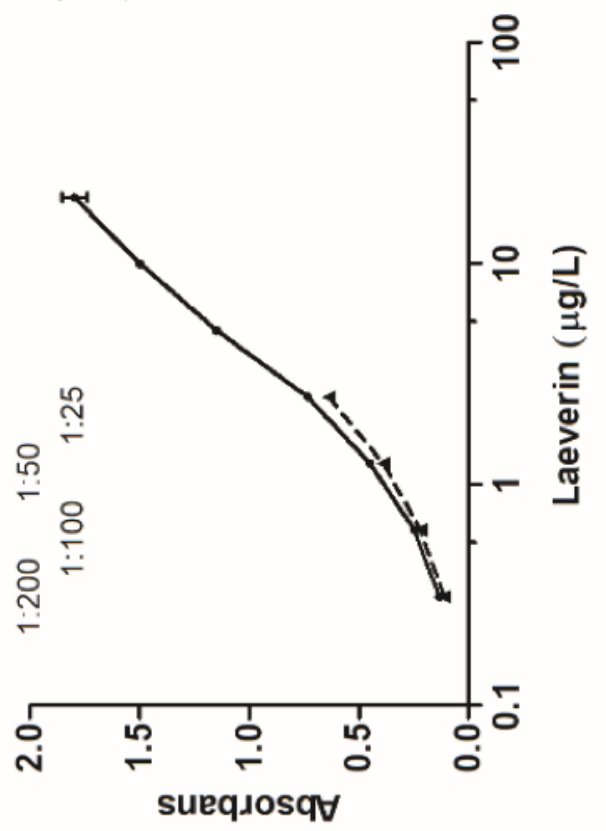



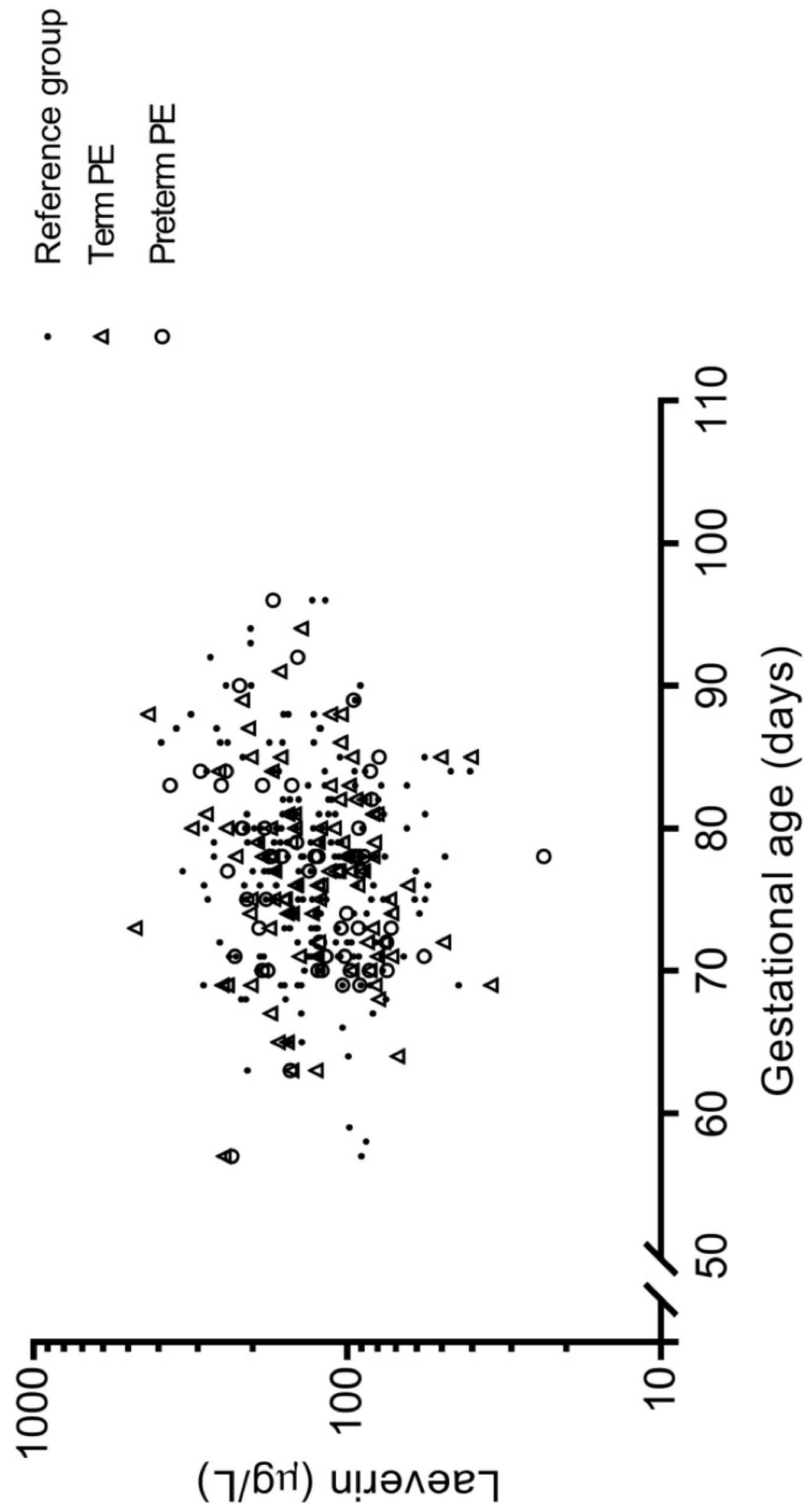